\title{
Exosomes: A Novel Biomarker for Bladder Cancer
}

\author{
Bin Li ${ }^{1,2}$, Wujian Deng ${ }^{3}$, Dunzhu Ciren ${ }^{4}$, Renlei Yuan ${ }^{4}$, Wei Qin ${ }^{4,5}$ and Xin Li ${ }^{* 6,7}$ \\ ${ }^{1}$ Department of Urinary surgery, the First People's Hospital of Foshan, China \\ ${ }^{2}$ Department of Urinary surgery, the People's Hospital of Linzhi, China \\ ${ }^{3}$ Department of General Surgery, the Second Affiliated Hospital of Guangzhou Medicial University, China \\ ${ }^{4}$ Department of Operative Dentistry and Endodontics, the People's Hospital of Linzhi, China \\ ${ }^{5}$ Guangdong Provincial Key Laboratory of Stomatology, Sun Yat-sen University, China \\ ${ }^{6}$ Department of Emergency, the People's Hospital of Linzhi, China \\ ${ }^{7}$ Department of Emergency Medicine, Department of Emergency and Critical Care Medicine, Guangdong Academy of Medical Sciences, China
}

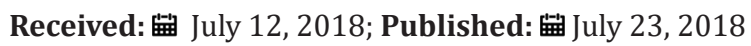

*Corresponding author: Xin Li, Department of Emergency, the People's Hospital of Linzhi, Xizang, 860000, P.R. China, Tel: 86-

18902233721; Email: drxinli@163.com

\begin{abstract}
Bladder cancer is one of the most common malignant tumors in the human body. At this stage, there is no significant progress in treatment. Therefore, early diagnosis of bladder cancer is particularly important. At present, the urine cytology screening, cystoscopy and pathological analysis are the most effective method for bladder cancer diagnosis. Due to the low specificity of urine exfoliated cells and cystoscopy is an invasive examination which is difficult for many patients to accept, there is an urgent need to find biomarkers of bladder cancer and to elucidate the mechanism of progression of bladder cancer for later treatment. Recently, some scholars have focused their attention on exosomes, which are a kind of nano-sized vesicle structure secreted by cells. They are distributed in various body fluids such as blood, saliva and urine. As an important class of intercellular communication molecules, exosomes contain a variety of biologically active components, which can play a regulatory role in the human body in a variety of ways. This paper is mainly about the introduction of bladder cancer exosomes.
\end{abstract}

Keywords: Bladder Cancer; Exosomes; Biomarker

Abbreviations: HDL: High-Density Lipoproteins; RISC: RNA-Induced Silencing Complex

\section{Introduction}

Bladder cancer is one of the 8 most common malignant tumors in the human body, and its incidence rate is the first in urological malignancies. Its causes mainly involve genetic factors, environmental factors, living habits and infections. Because the incidence of bladder cancer is concealed, there is no special discomfort in the early stage and the recurrence rate is high [1]. Therefore, early detection and early treatment are advocated. Exosomes have been in existence for more than 30 years since their discovery and were originally thought to be the "waste" of cells [2] It was later discovered that exosomes are secretory macrovesicles that are functionally active and capable of intercellular communication [3]. At present, exosomes are found in many types of cells, and tumor cell derived exosome has become a research hotspot in the field of cancer. Studies have shown that differential proteins in urine exosomes of patients with bladder cancer can be used for early diagnosis of bladder cancer, and it is confirmed that there is a high correlation. These have brought new ideas to the early detection of bladder cancer.

\section{Protein in Exosomes may be an Important Indicator for Early Diagnosis of Bladder Cancer}

Exosomes are vesicle-like bodies that are actively secreted extracellularly by a variety of living cells. Proteomics is a systematic biology study of processes such as disease development and cellular metabolism at the protein level. To elucidate the mechanism of exosomes in forming and maintaining microenvironment of tumor cell survival, proliferation and metastasis, and inducing tumor cell proliferation, we can study the tumor cells derived exosomes by proteomics technology platform, some progress has been made in the field of tumor biomarkers, which has shown good prospects for the application of exosomes in clinical diagnosis and treatment. The basic research found that: the exogenous proteome of the rat lung tissue and liver tissue metastasis T24 allele SLT4 and FL3 cell lines compared with non-metastatic bladder cancer T24 cells, Vimentin, liver cancer-derived growth factor and casein kinase II $\alpha$, membrane raft protein A2 and other epithelial mesenchymal cell migrationrelated proteins are only present in two exosomes with metastatic 
cell lines [4], revealing that malignant tumor-derived exosomes play a role in maintaining the function of tumor cells invading the microenvironment. Some scholars have shown that exosomes secreted by bladder cancer cells contain some important proteins that affect tumor progression [4-7], and these exosomes inhibit apoptosis of bladder cancer cells through Akt and ERK metabolic pathways [8].

\section{MicroRNAs (miRNAs) in Exosomes can be a Therapeutic Target for Bladder Cancer}

MicroRNAs (miRNAs) are endogenous, single-stranded, noncoding microRNAs that specifically bind to the 3' untranslatable region ( $3^{\prime} U T R$ ) of a target $m R N A$ to induce degradation or inhibit translation, thereby effectively silencing protein expression of the target gene at the post-transcriptional level. Because miRNAs can regulate the expression of oncogenes or tumor suppressor genes in the above manner, they are widely implicated in the development of tumors. MiRNAs are typically 19 to $23 \mathrm{nt}$ in length and have long been taken into account a by-product of transcription. Current research indicates that miRNAs have important functional significance in physiological and pathological processes, and involved in almost all related processes of tumorigenesis, such as cell growth, differentiation, proliferation, angiogenesis, apoptosis, invasion and metastasis [9]. Recent studies have shown that miRNAs released by active cells can be measured in body fluids. These miRNAs are collectively referred to as extracellular miRNAs and are usually stably present in microvesicles, especially exosomes. Exosomes is a major protective mechanism of extracellular miRNAs and plays an important role in intercellular communication [10], which is used as a promising disease diagnosis and prognostic biomarker in urinary malignancy. Intracellular miRNAs is divided into two different subpopulations, endogenous and exogenous. In the cytosol, pre-miRNAs were further trimmed by Dicer into mature miRNAs with a length of 19 to $23 \mathrm{nt}$ [11].

The miRNAs produced by the above pathways constitute most of the miRNAs in the cell. Another source of intracellular miRNAs is the extracellular environment. Researchers generally believe that cells may release complexes containing miRNAs into biological fluids such as blood, saliva, urine, and milk [12]. These complexes include miRNAs-binding proteins [13] and high-density lipoproteins (HDL) [14], or microvesicles. When these complexes circulate in the blood, they may be captured by distant cells, enabling miRNAs-mediated intercellular communication. Mature mi RNAs have a significant impact on cellular function by modulating target mRNAs. The miRNAs form a RNA-induced silencing complex (RISC) with Ago 2 and then bind to the 3' non-coding region (3'UTR) of the target mRNAs in a complete or incomplete pairing manner. If miRNAs are perfectly paired with mRNAs, the target mRNAs is cleaved by endonucleases; if they do not match exactly, translation of the target mRNAs is inhibited. It estimates that approximately 1000 miRNAs regulate more than $30 \%$ of human genes [15]. Studies have shown that exosomes are released in large quantities in biological fluids, including blood, urine, cerebrospinal fluid, malignant hydrothorax and ascites, amniotic fluid and synovial fluid [16]. Exosomes are capable of carrying miRNAs circulating in the blood for long-distance intercellular communication. In view of this we can extract exosomal miRNAs from various body fluids as a diagnostic and prognostic marker for disease.

Ideal biomarkers for diagnosis and prognosis should comply with the following criteria: easy to obtain through non-invasive methods, high sensitivity and specificity, ability to detect early stages of disease, longer half-life of samples, and fast and accurate detection. Among them, the non-invasive method is the most basic acquisition requirement. The common biological fluids collected by non-invasive methods are serum and plasma, and circulating miRNAs have attracted much attention in recent years. Compared with mRNAs, circulating miRNAs are stable enough to stand physical degradation such as storage and freeze-thaw cycles, and are also resistant to biochemical degradation such as ribonuclease (RNAse) and RNAseA in vitro [17]. In the blood-rich environment of RNAse, many mechanisms protect circulating miRNAs, of which exosomes are thought to be a major protective mechanism [18]. The outer membrane of the exosomes provides comprehensive RNA protection, which will be separated from the extracellular environment that is easily digested. Another common biological fluid collected in a non-invasive manner is urine. Urine is not only easy to obtain, but also easy to collect, non-invasive, and large quantity. Therefore, urine is an ideal liquid for reflecting potential miRNAs in urinary system diseases. Because urine passes through the urinary malignant tumor before excretion, its miRNAs often reflect the state of the disease. The protective mechanisms of miRNAs present in urine are similar to those of circulating miRNAs, but due to the complex composition of urine and the large amount of RNAse activity in the urinary system, naked miRNAs in urine are more susceptible to degradation, so the research stems from exosome miRNAs are more realistic. Urine exosomes can be derived from urinary tract tumors (such as prostate cancer, bladder cancer, and kidney cancer), normal urinary epithelium, glomeruli, or renal tubules. In a kidney disease study, RNase A was used to deal with urine sediment before urinary exosomal RNA extraction. The results showed that both AQP2 and CD24 were stably expressed in the treated and untreated groups, and there were no significantly difference of the Ct values were between the two groups [19].

In another study, RNA was extracted from whole urine, urinary deposit, cell-free urine, exosomal urine supernatant, and urine exosomes, and miRNAs of length $<200 \mathrm{nt}$ were analyzed. As a result, enriched intact miRNAs were found only in urine exosomes [20]. Although a certain number of miRNAs are contained in the urine cell pellet, most of them have degraded. Further studies showed that 184 exosomal-associated miRNAs were detected by highthroughput deep sequencing in 3 urine samples from separate subjects, and only 7 of these miRNAs were shared with cell-free urine. The above results indicates that urine exosomes are not only rich in intact miRNAs, but also have a good protective effect on RNAs. Most of the miRNAs markers for the diagnosis and prognosis of bladder cancer use urine. Hanke et al. [21] identified 157 miRNAs expression profiles in the urine of bladder cancer patients, and identified a significant increase in the expression levels of miRNA-126, miRNA-182 and miRNA-199a, as well as miRNA-126 in urine. 
The ratio of miRNA-152 was able to detect bladder cancer, with sensitivity, specificity, and area under the curve of $72 \%, 82 \%$, and 0.768 , respectively. Recently, studies have found that miRNA-145, miRNA-143, and miRNA-125b are significantly down-regulated in bladder cancer tissues, but miRNA-183, miRNA-96, miRNA-175p, and miRNA-20a are significantly up-regulated [22], another study showed that miRNA-96 and miRNA-183 in urine have high sensitivity and specificity in distinguishing bladder cancer from non-cancer patients, miRNA-96 is $71.0 \%$ and $89.2 \%$, miRNA- 183 was $74.0 \%$ and $77.3 \%$, respectively. In this study, the expression levels of miRNA-96 and miRNA-183 in urine of bladder cancer patients were significantly higher than those in healthy controls, but the expression levels of the above two miRNAs in urine were significantly decreased after surgery.

When miRNA-96 bind to urine cytology, the sensitivity of urine cytology can be increased from $43.6 \%$ to $78.2 \%$, so miRNA-96 can be used as an excellent diagnostic marker [23]. Wang et al. [24] performed urine microcentrifugation on urine sediment and supernatant of bladder cancer patients, and miRNA-200 family, miRNA-192 and miRNA-155 in urine sedimentation. Low expression of mi RNA-192 in urine supernatant, but high expression of mi RNA-155. The expression of the miRNA-200 family was increased in urine sediment after tumor tissue resection in this study. The above findings indicates the potential of miRNAs as a non-invasive biomarker for bladder cancer in urine. Because of the different outcomes of the study subjects and methods, patient recruitment, sample collection, miRNAs extraction, and quantitative standardization are essential. The current study is generally based on small sample patients, and large-scale multicenter studies are urgently needed.

\section{Exosomes can be Used as an Important Entry Point to Study the Microenvironmental Changes of Bladder Cancer}

Exosomes are a new information transmission carrier. The exchange of cells with the external environment is not only active transport, passive transport [25], but also other molecular mechanisms. Among them, membrane transport is particularly prominent, especially exosome regulated substances. Transportation is getting more and more attention [26]. Exosomes are not just involved in the transport of substances, but also play an important role in cell-cell or intracellular communication [27], such as mediating the transfer of tumor cell antigens, activation of dendritic cells [28]. The information exchange mechanism of exosomes [29] mainly includes:

a) Exosomes recognize and fuse with target cells through their membrane proteins;

b) Lipids contained in exosomes can be transported to recipient cells via Notch signal. The pathway promotes apoptosis of cells;

c) Exosome-derived transcription factor receptors are involved in regulating the transcription of recipient cells;

d) Exosome-derived microRNAs can be involved in binding to RNA-induced silencing complex (RISC).
After post-transcriptional regulation of somatic cells, tumor cells promote the proliferation and invasion of tumor cells through information exchange with their own microenvironment [30], and exosomes can actas such information carriers. When a gene mutation occurs in a cell, the exosomes transmit this mutation information to other normal cells through membrane fusion, resulting in activation of the proto-oncogene, expression of anti-apoptotic genes, and an increase in anchor-independent growth ability. To make tumor cells obtain the characteristics of hyperplasia [31]. Tumor cell-derived exosomes promote the immune escape of tumor cells by directly inhibiting immune cells or regulating the expression of related cytokines, and creating conditions for tumor cells to grow in vivo. For example, bladder cancer cell-derived exosomes can inhibit apoptosis in bladder cancer cells [32]. Despite the fact that surgery is the mainstay, combined with comprehensive treatment, many tumors have achieved good therapeutic effects, but the prognosis of many solid tumors is still poor, because the occurrence, evolution and recurrence mechanism of tumors are still unclear. Studies have shown that tumor stem cells (CSCs) are found in tumors, which are the root cause of tumorigenesis, recurrence and metastasis. Therefore, CSCs are expected to be an ideal target for cancer treatment, and in-depth study of its biological characteristics is of great significance in improving the level of tumor prevention and treatment. CSCs possess certain biological properties, including self-renewal capacity, high proliferative activity, and multidirectional differentiation potential, which can be called "dry" and are key factors in the malignant behavior of CSCs. After exosomes are secreted from tumor cells, the microenvironment before tumor metastasis is regulated by secretory proteins in a novel environment, and this microenvironmental change will significantly affect the dryness of tumor cells [33]. A series of studies have shown that exosomes can reflect the hypoxic state of tumor cells [34], and the hypoxic microenvironment maintains the self-renewal and multidirectional differentiation potential of tumor cells. Exosomes affect the dryness of tumor cells by up-regulating various signaling pathways such as Notch and Wnt. As mentioned above, a large body of evidence indicates that exosomes promote tumorigenesis and development. However, the mechanic is still unclear and large-scale clinical trials are needed to clarify their intrinsic links. Application techniques of exosomes will bring revolutionary progress in the diagnosis and treatment of bladder cancer in the future.

\section{References}

1. Resnick MJ, Chang SS (2013) Optimizing outcomes for octogenarians with invasive bladder cancer: one size does not fit all. Urologic Oncology 31(1): 1-4.

2. Corrado C, Raimondo S, Chiesi A, Ciccia F, De Leo G, et al. (2013) Exosomes as intercellular signaling organelles involved in health and disease: basic science and clinical applications. Int J Mol Sci 14(3): 5338-5366.

3. Dsouza Schorey C, Clancy JW (2012) Tumor-derived microvesicles: shedding light on novel microenvironment modulators and prospective cancer biomarkers. Genes Dev 26(12): 1287-1299.

4. Welton JL, Khanna S, Giles PJ, Brennan P, Brewis IA, et al. (2010) Proteomics analysis of bladder cancer exosomes. Molecular and Cellular Proteomics 9(6): 1324-1338.

5. Pisitkun T, Johnstone R, Knepper MA (2006) Discovery of urinary biomarkers. Molecular and Cellular Proteomics 5(10): 1760-1771. 
6. Chen CL, Lai YF, Tang P, Chien KY, Yu JS, et al. (2012) Comparative and targeted proteomic analyses of urinary microparticles from bladder cancer and hernia patients. Journal of Proteome Research 11(12): 56115629.

7. Yang L, Wu XH, Wang D, Chun-Li Luo, Li Xue Chen, et al. (2013) Bladder cancer cell-derived exosomes inhibit tumor cell apoptosis and induce cell proliferation in vitro. Molecular Medicine Reports 8(4): 1272-1278.

8. Yang Y, Kang CS (2011) Recent advances in the research on the relationship between mi RNA-21 and cancer. Chin J Clin Oncol 38(6): 357-360.

9. Huang X, Liang M, Dittmar R, Liang Wang (2013) Extracellular micro RNAs in urologic malignancies: chances and challenges. Int J Mol Sci 14(7): 14785-14799.

10. Catton JW, Alcaraz A, Bjartell AS, De Vere White R, Evans CP, et al. (2011) Micro RNA in prostate, bladder, and kidney cancer: a systematic review. Eur Urol 59(5): 671-681.

11. Vlassov AV, Magdaleno S, Setterquist R, Conrad R (2012) Exosomes: current knowledge of their composition, biological functions, and diagnostic and therapeutic potentials. Biochim Biophys Acta 1820(7): 940-948.

12. Arroyo JD, Chevillet JR, Kroh EM, Ruf IK, Pritchard CC, et al. (2011) Argonaute 2 complexes carry a population of circulating micro RNAs independent of vesicles in human plasma. Proc Natl Acad Sci U S A 108(12): 5003-5008.

13. Vickers KC, Palmisano BT, Shoucri BM, Shamburek RD, Remaley AT (2011) Micro RNAs are transported in plasma and delivered to recipient cells by high-density lipoproteins. Nat Cell Biol 13(4): 423-433.

14. Schaefer A, Stephan C, Busch J, Yousef GM, Jung K (2010) Diagnostic, prognostic and therapeutic implications of micro RNAs in urologic tumors. Nat Rev Urol 7(5): 286-297.

15. Simpson RJ, Jensen SS, Lim JW (2008) Proteomic profiling of exosomes: current perspectives. Proteomics 8(19): 4083-4099.

16. Reid G, Kirschner MB, van Zandwijk N (2011) Circulating micro RNAs: Association with disease and potential use as biomarkers. Crit Rev Oncol Hematol 80(2): 193-208.

17. Huang X, Yuan T, Tschannen M, Zhifu Sun, Howard Jacob, et al. (2013) Characterization of human plasma-derived exosomal RNAs by deep sequencing. BMC Genomics 14: 319.

18. Lv LL, Cao Y, Liu D, Xu M, Liu H, et al. (2013) Isolation and quantification of micro RNAs from urinary exosomes/microvesicles for biomarker discovery. Int J Biol Sci 9(10): 1021-1031.

19. Cheng L, Sun X, Scicluna BJ, Coleman BM, Hill AF (2014) Characterization and deep sequencing analysis of exosomal and non-exosomal mi RNA in human urine. Kidney Int 86(2): 433-444

20. Hanke M, Hoefig K, Merz H, Feller AC, Kausch I, et al. (2010) A robust methodology to study urine micro RNA as tumor marker: micro RNA-
126 and micro RNA-182 are related to urinary bladder cancer. Urol Oncol 28(6): 655-661.

21. Yoshino H, Seki N, Itesako T, Chiyomaru T, Nakagawa $M$, et al. (2013) Aberrant expression of microRNAs in bladder cancer. NatureReviewsUrology 10(7): 396-404.

22. Yamada Y, Enokida H, Kojima S, Kawakami K, Chiyomaru T, et al. (2011) miR-96 and miR-183 detection in urine serve as potential tumor markers of urothelial carcinoma: correlation with stage and grade, and comparison with urinary cytology. Cancer Sci 102(3): 522-529.

23. Wang G, Chan ES, Kwan BC, , Li PK, Yip SK, et al. (2012) Expression of micro RNAs in the urine of patients with bladder cancer. Clin Genitourin Cancer 10(2): 106-113.

24. Hsu C, Morohashi Y, Yoshimura S, Manrique Hoyos N, Jung S, et al. (2010) Regulation of exosome secretion by Rab35 and its GTPaseactivating proteinsTBC1D10A-C. J Cell Biol 189(2): 223-232.

25. Sugano K, Kansy M, Artursson P, Avdeef A, Bendels S, et al, (2010) Coexistence of passive and carrier-mediated processes in drug transport. Nat REV Drug Discov 9(8): 597-614.

26. EL Andaloussi S, Mäger I, Breakefield X O,Wood MJ (2013) Extracellularvesicles: biology and emerging therapeutic opportunities. Nat Rev Drug Discov 12(5): 347-357.

27. Staals RH, Pruijn GJ (2010) The human exosome and disease. Adv Exp Med Biol 702: 132-142.

28. Liu XT, Wang SW, Liu J (2013) Exosomes: The Novel Vehicles for Intercellular Communication. Prog Biochem Biophys 40( 8): 719-727.

29. Kahlert C, Kalluri R (2013) Exosomes in tumor micro environment influence cancer progression and metastasis. J Mol Med( Berl) 91(4): 431-437.

30. Al Nedawi K, Meehan B, Micallef J, Lhotak V, May L, et al. (2008) Intercellular transfer of the oncogenic receptor EGFRv III by microvesicles derived from tumour cells. Nat Cell Biol 10(5): 619-624.

31. Yang L, Wu X H, Wang D, Luo CL, Chen LX (2013) Bladder cancer cell-derived exosomes inhibit tumor cell apoptosis and induce cell proliferation in vitro. Mol Med Rep 8(4): 1272-1278.

32. Rehn M, Olsson A, Reckzeh K, Diffner E, Carmeliet P, et al. (2011) Hypoxic induction of vascular endothelial growth factor regulates murine hematopoietic stem cell function in the low-oxygenic niche. Blood 118(6): 1534-1543.

33. Kucharzewska P, Christianson HC, Welch JE, Katrin J Svensson, Erik Fredlund, et al. (2013) Exosomes reflect the hypoxic status of glioma cells and mediate hypoxia-dependent activation of vascular cells during tumor development. Proc Natl Acad Sci U S A 110(18): 7312-7317.

34. Takebe N, Harris PJ, Warren RQ, Ivy SP (2011) Targeting cancer stem cells by inhibiting Wnt, Notch, and Hedgehog pathways. Nat Rev Clin Oncol 8(2): 97-106.

\section{ISSN: 2574-1241}

DOI: 10.26717/BJSTR.2018.07.001458

Xin Li. Biomed J Sci \& Tech Res

This work is licensed under Creative

Commons Attribution 4.0 License

Submission Link: https://biomedres.us/submit-manuscript.php

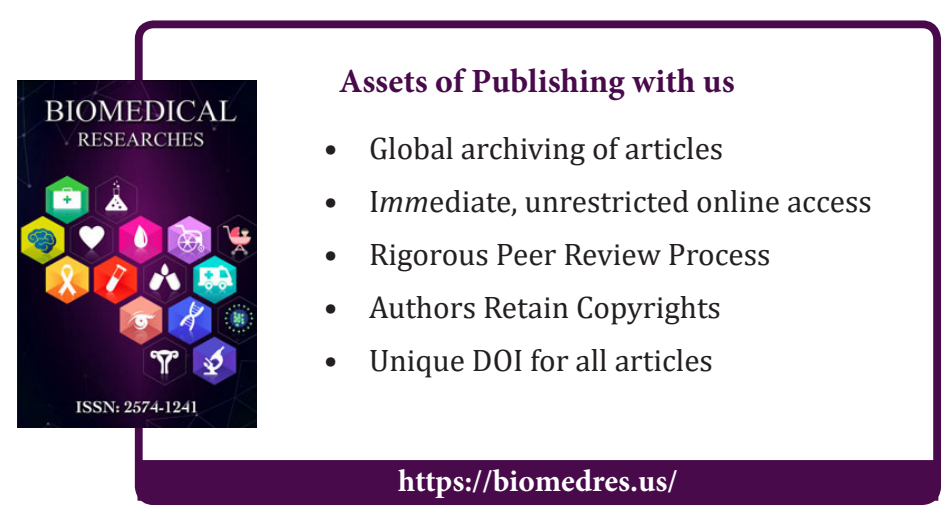

